\title{
Calculation of Phenomenological Coefficients by Monte Carlo Computer Simulation Methods
}

\author{
I.V. Belova ${ }^{1, a}$ M.J. Brown ${ }^{1, b}$ and G.E. Murch ${ }^{1, c}$ \\ ${ }^{1}$ Diffusion in Solids Group, School of Engineering, The University of Newcastle, Callaghan, NSW \\ 2308, Australia \\ arina.Belova@newcastle.edu.au, bMandy.Brown@studentmail.newcastle.edu.au, \\ 'Graeme.Murch@newcastle.edu.au
}

Keywords: Phenomenological coefficients, Monte Carlo, lattice gas, correlation effects

\begin{abstract}
In this paper we first review the principal indirect and direct Monte Carlo methods for calculating the Onsager phenomenological transport coefficients in solid state diffusion. We propose a new Monte Carlo method that makes use of a steady state calculation of a flux of atoms that is driven by a difference in chemical potential of the atoms between a source and a sink plane. The method is demonstrated for the simple cubic one component lattice gas with nearest neighbour interactions. The new method gives results in good agreement with a Monte Carlo method based on Einsteinian expressions for the phenomenological coefficients.
\end{abstract}

\section{Introduction}

It has long been appreciated that Fick's First Law is frequently insufficient as a condition for attaining equilibrium of a given species $i$ because it does not recognize all driving forces, direct and indirect, acting on $i$. The Onsager flux equations of irreversible processes achieve this through the postulate of linear relations between the fluxes and the driving forces, see for example [1,2]:

$$
J_{i}=\sum_{j} L_{i j} X_{j}
$$

where $J_{i}$ is the flux of species $i$, the $L_{i j}$ are the phenomenological transport coefficients and the $X_{i}$ are the driving forces and the summation is over all species including $i$. The importance of the phenomenological coefficients or $\boldsymbol{L}$ matrix stems from their independence of driving force. The Onsager flux equations have been used very extensively in theoretical treatments of collective diffusion problems such as chemical diffusion and ionic conductivity especially in multicomponent systems. Phenomena such as the vacancy-wind effect [3] and correlation effects owe their origins to diagonal and, especially, non-zero off-diagonal phenomenological coefficients. In one-component systems there are also correlation effects (variously called 'physical', 'conductivity' or 'collective' correlation effects) that are incorporated in the (single) phenomenological coefficient [4].

In the solid state, where the hopping model (the atoms thermalize with their surroundings between hops or jumps) is extremely commonly used, it is usual to express phenomenological coefficients as the product of a correlated part and an uncorrelated part (the latter is essentially the jump frequency). The correlated part consists of a correlation function; more commonly termed a collective correlation factor. The calculation of a phenomenological coefficient is seen to be analogous to the calculation of a tracer diffusion coefficient which requires the calculation of a correlated part (the well-known tracer correlation factor) and an uncorrelated part (the jump frequency). The phenomenological coefficient is thus partitioned as [2]:

$$
\begin{aligned}
& L_{i i}=f_{i i} a^{2} C_{i} n_{i} / 6 k T t, \\
& L_{i j}=f_{i j}^{(i)} a^{2} C_{i} n_{i} / 6 k T t,
\end{aligned}
$$

or, alternatively: 


$$
L_{i j}=f_{i j}^{(j)} a^{2} C_{j} n_{j} / 6 k T t
$$

where $C_{i}=c_{i} N / V\left(c_{i}\right.$ is the site fraction of the $i$ species and $N$ is the total number of lattice sites in the system of total volume $V$ ) and $n_{i}$ is the number of jumps of species $i$ in time $t$ and the $f_{i j}$ are the collective correlation factors and $k$ and $T$ have their usual meanings.

Over the past thirty years there have been many calculations of the phenomenological coefficients; we are specifically referring here to calculations (often called diffusion kinetics calculations) of the correlated part of the phenomenological coefficient not the uncorrelated or jump frequency part, of which there are, of course, a great many stand-alone calculations. The calculations of the correlated part fall into two groups: analytical calculations and Monte Carlo computer simulations. The Monte Carlo simulations have frequently been used to test directly the results from the analytical calculations but, in the early years, the Monte Carlo simulations tended to have an independent existence. The analytical calculations, which make use of a very wide range of methods, have tended to concentrate on the five-frequency model for dilute systems [5] and the Ising alloy model [6,7] and the non-interacting model (the random alloy model) [8,9] for concentrated systems. The Monte Carlo simulations have also generally concentrated on the same models. The focus of the present paper is the Monte Carlo simulations. First, we present a review of the principal Monte Carlo methods that have been proposed for calculating, either indirectly and directly, the phenomenological coefficients. Next, we propose a new direct method based on the calculation of a steady state flux in a chemical potential gradient. This method is demonstrated with a one-component lattice gas.

\section{Review of Monte Carlo calculations of the phenomenological coefficients}

Indirect Methods. The indirect methods for gaining access to phenomenological coefficients in the solid state seem only to have been employed so far for the one-component lattice gas. They effectively make use of the following relationship between the collective or chemical diffusion coefficient $D_{A}$ in the one-component $(A)$ lattice gas and the corresponding phenomenological coefficient $L_{A A}$ :

$$
D_{A}=\frac{k T L_{A A}}{C_{A}}\left(\frac{\partial(\mu / k T)}{\partial \ln c_{A}}\right),
$$

where $\mu$ is the lattice gas chemical potential. The factor in parentheses is the thermodynamic factor. A calculation of the phenomenological coefficient performed in this way thus requires the calculation of both the collective diffusion coefficient (which contains the collective correlation factor $f_{A A}$ ) and the thermodynamic factor.

We describe two methods, both of which use Fick's Second Law in order to gain access to the collective diffusion coefficient. Both are well-suited to the one-component lattice gas but are not readily extended to binary systems since they depend on a change in composition that in the binary system would probably be expressible only in terms of a combination of phenomenological coefficients. In the first method, a periodic lattice is prepared in a way that represents, on a hydrodynamic scale, a constant composition $\mathrm{c}_{\mathrm{A}}^{\mathrm{A}}$ plus a cosine composition profile in, say, the $x$ direction [10]:

$$
c_{A}(x)=c_{\mathrm{A}}^{\prime}+\delta c_{\mathrm{A}} \cos (k x),
$$

where $k=2 \pi / \lambda$ and $\lambda$ is the wavelength of the composition disturbance. Strictly, the above composition profile should be carefully prepared in such a way that the composition on each plane itself is locally equilibrated but this does not usually done. Assuming the decay with time $t$ of the cosine composition profile is governed by the Diffusion Equation (Fick's Second Law), i.e. it is simply a first-order relaxation, the amplitude $\delta c_{\mathrm{A}}$ then simply decays as $\exp \left(-D_{\mathrm{A}} k^{2} t\right)$ and one can 
immediately extract the collective diffusion coefficient. In general, the collective diffusion coefficient is a function of composition (an exception is when the atoms are non-interacting) and so the composition disturbance needs to be relatively close to that of the background composition so that this composition dependence is not neglected. This is probably a shortcoming of this method. The thermodynamic factor itself can be obtained independently by way of a numerical derivative of chemical potential versus composition data obtained using either the petit canonical $(N, V, T)$ ensemble and the particle insertion method [11,12] or the grand canonical $(\mu, V, T)$ ensemble [12].

In the second method, the periodic system is prepared, again on a hydrodynamic scale, with two compartments at different compositions in a direct analogue of a standard experimental interdiffusion couple [13]. The composition profile after diffusion for a given time $t$ is established and the collective diffusion coefficient is determined. If the compositions of the two compartments are relatively close, then an error function type solution can be employed. On the other hand, if the compositions are very different, the well-known Boltzmann-Matano analysis can then be used for including directly the dependence of the collective diffusion coefficient on composition. Again, the thermodynamic factor can be determined independently as above in order to gain access to the phenomenological coefficient itself.

In principle, both of these methods could take the system outside the linear response region especially when the initial composition gradient is very large but this does not seem to have been tested. Neither of these indirect methods appears to be used any longer for calculation phenomenological coefficients but they have an important pedagogical use.

Direct Methods. The methods described in this section enable the phenomenological coefficients to be calculated directly in the simulation. The first direct method makes use of an external field $E$ acting on a homogeneous system with periodic boundaries. The field biases the jumps in, say, the $x$ direction [14]. The driving forces $X_{i}$ in the Onsager flux equations (Eq. 1) can then be written conveniently simply as $-q_{i} E$ where $q_{i}$ is a hypothetical 'charge' on species $i$ (used purely for the purposes of the calculation). Calculation of the flux(es) resulting from the field enables the phenomenological coefficients directly. The driving forces can be switched on and off according to the charge on the species. In, for example, a binary alloy system $A B$, if $A$ 'feels' the field $\left(q_{A} \neq 0\right)$ but $B$ does not $\left(q_{B}=0\right)$, then the drift of $A$ can be processed immediately to give $L_{A A}$ and the indirect drift of $B$ can be processed immediately to give $L_{A B}$. On the other hand, if $B$ 'feels' the field $\left(q_{B} \neq 0\right)$ but $A$ does not $\left(q_{A}=0\right)$, then the drift of $B$ gives $L_{B B}$ and the drift of $A$ gives $L_{A B}$. Since the field is wrapped around the simulated lattice via the periodic boundary in the $x$ direction, there is no segregation of $A$ or $B$ as a result of the field. The external field method has been used many times in both the one-component lattice gas and various binary alloy systems; see, for example [14-17]. It was used to demonstrate, for the first time, the validity of the vacancy-wind effect in the fivefrequency model [17] and collective correlation in the one-component lattice gas [14]. For the relatively small lattice systems generally dealt with, it is necessary to impose rather large external fields to obtain fluxes that are distinguishable from the background noise. This can make the corresponding results for the phenomenological coefficients slightly field-dependent (in effect the field can enter the Hamiltonian of the model). Accordingly, it is usually necessary to gather results at a range of field strengths and then extrapolate to zero electric field.

Although frequently used in the 1970s and 1980s, the external field method is now primarily used for pedagogical purposes. It has now largely been replaced in research contexts by a second direct method based on very important expressions derived by Allnatt [18] for the phenomenological coefficients. Allnatt showed that the phenomenological coefficients can be expressed in a form analogous to the Einstein Equation for the tracer diffusion coefficient:

$$
D^{*}=<\boldsymbol{R}^{2}>/ 6 t \text {, }
$$

where $\boldsymbol{R}$ is the displacement of a given atom in time $t$ and the Dirac brackets refer to a large number of atoms. Allnatt's analogous expressions for the phenomenological coefficients are: 


$$
\begin{aligned}
& L_{i i}=<\boldsymbol{R}_{i} \boldsymbol{R}_{i}>/ 6 t V k T, \\
& L_{i j}=<\boldsymbol{R}_{i} \boldsymbol{R}_{i}>/ 6 t V k T,
\end{aligned}
$$

where $\boldsymbol{R}_{i}$ and $\boldsymbol{R}_{i}$ are the collective displacements of species $i$ and species $j$ in the time $t$. The collective displacement of a species is, in effect, the displacement of the centre of mass of that species. In a computer simulation, say of a random binary alloy, one would have a periodic lattice of volume $V$ containing $N$ sites on which the two species $A$ and $B$ are (say) randomly distributed. One allows diffusion by vacancies to occur for some time $t$ and then simply calculates the displacements of the centres of mass of the $A$ atoms and of the $B$ atoms in that time. This entire procedure is repeated a large number of times, typically of the order of 10000 , in order to produce the ensemble average $<>$. Although substantially more computer time is required for this method compared with the first direct method, in our opinion, the principal advantages are the simplicity of programming and that no extrapolation to zero field is required. Importantly, the detailed structure of the collective correlation factors themselves can be readily exposed if required. For example, the diagonal collective correlation factors (the collective correlation factors of the diagonal phenomenological coefficients) are readily decomposed to the following expression [19] (which is quite analogous to the well-known tracer correlation factor expression [3]):

$$
f_{i i}=1+2 \sum_{m=1}^{\infty}<\cos \theta_{i i}^{(m)}>
$$

where $\left\langle\cos \theta_{i i}^{(m)}>\right.$ is the average of the cosine of the angle between some jump of an atom of species $i$ and the $m$ 'th succeeding jump of the same or any other atom of species $i$. These cosines can be calculated readily during the simulation and averaged appropriately.

\section{A New Simulation Method for Calculating the Phenomenological Coefficient}

Although most driving forces for diffusion in real materials are internal gradients of the chemical potentials of the species present, a method has not previously been proposed to use such internal chemical potential gradients in a computer simulation. In this section, we describe a new calculation of the phenomenological coefficient $L_{A A}$ in the one-component lattice gas using a steady-state atom flux resulting from a chemical potential gradient of the atoms. Since there is considerable confusion in the literature about the various chemical potentials in the lattice gas and how they might enter into the Onsager flux equations, we first provide some detail on these points.

Chemical Diffusion in the One-Component Lattice Gas. Consider a lattice gas system consisting of one type of atom $(A)$ and vacancies $(V)$, where the atoms can exchange with nearest neighbour vacancies. Non-trivial interactions between the atoms are assumed. The Onsager flux equations can be written out in the usual way [2], (see Eq. 1):

$$
\begin{aligned}
& J_{A}=L_{A A} X_{A}+L_{A V} X_{V} \\
& J_{V}=L_{A V} X_{A}+L_{V V} X_{V}
\end{aligned}
$$

Since atoms and vacancies exactly mirror each other's motion then obviously $J_{A}=-J_{V}$. It is then straightforward to show from Eqs. 8 that:

$$
J_{A}=L_{A A}\left(X_{A}-X_{V}\right)
$$

In the absence of external forces such as electric fields, the driving forces are the negative of the chemical potential gradients, written as $X_{i}=-\nabla \mu_{i}$ where $\mu_{i}$ is the chemical potential of species $i$. These chemical potentials are defined in the canonical ensemble as:

$$
\mu_{i}=\left(\frac{\partial A}{\partial N_{i}}\right)_{T, N_{\alpha \neq i}},
$$


where $A$ is the Helmholtz free energy, $N_{i}$ is the number of $i$ entities (atoms or vacancies).

We then have the important result that:

$$
J_{A}=-L_{A A} \nabla\left(\mu_{A}-\mu_{V}\right)
$$

or,

$$
J_{A}=-L_{A A} \nabla \mu
$$

The quantity $\mu$ is frequently referred to as the (one-component) lattice gas chemical potential and was introduced above without comment in Eq. 3. This chemical potential is formally defined as:

$$
\mu=\left(\frac{\partial A}{\partial N_{i}}\right)_{T, V}
$$

Note, the two chemical potentials $\mu$ and $\mu_{A}$ are not equal. But at very low compositions of $A$ atoms these two chemical potentials do converge. For the case of non-interacting atoms (but including self-blocking) $\mu$ is given by what is sometimes called in adsorption physics the Langmuir isotherm $\mu / k T=\ln \left(c_{A} /\left(1-c_{A}\right)\right.$. For the same conditions we have that $\mu_{A} / k T=\ln c_{A}$ (and $\left.\mu_{V} / k T=\ln c_{V}\right)$.

Writing the Gibbs-Duhem relation:

$$
c_{A} d \mu_{A}+c_{V} d \mu_{V}=0 \text {, }
$$

where $c_{\mathrm{V}}$ is the site fraction of vacancies, and on substituting $c_{\mathrm{A}}=1-c_{\mathrm{V}}$, we have that:

$$
\begin{aligned}
d \mu_{A} & =c_{V}\left(d \mu_{A}-d \mu_{V}\right) \\
& =c_{V} d \mu
\end{aligned}
$$

As mentioned above in connection with Eq. 6, the phenomenological coefficient $L_{\mathrm{AA}}$ can be written in an Einsteinian form which can then be expanded in the well-known hopping model framework to give:

$$
\begin{aligned}
L_{A A} & =<\boldsymbol{R}_{\mathrm{A}}^{2}>/ 6 V k T t, \\
& =z p_{\mathbf{A V}} w_{A} a^{2} C_{A} f_{A A} / 6 k T,
\end{aligned}
$$

where $\boldsymbol{R}_{\mathrm{A}}$ is the collective displacement of the species $A$ in time $t$ in the volume $V, p_{A V}=p_{A V}\left(c_{A}\right)$ is the average vacancy availability factor, $w_{A}=w_{A}\left(c_{A}\right)$ is the average exchange frequency of an $A$ atom with a vacancy, $a$ is the jump distance, $f_{A A}$ is the collective correlation factor (this is very weakly dependent on composition and interactions above the order/disorder and critical temperatures in the nearest neighbour interacting lattice gas). It is worth noting that over the years the collective correlation factor $f_{A A}$ has variously been called the 'physical correlation factor' or the 'conductivity correlation factor' and has often been given the symbol $f_{I}$. The factor $f_{\mathrm{AA}}$ is the correlation factor of the centre of mass of the $A$ atoms and is the correlated part of the single phenomenological coefficient $L_{A A}$. Since the $A$ atoms and vacancies exactly mirror each other's motion then there is a correlation factor for the vacancies $f_{V V}$ but this carries no new information since it must always simply be equal to $f_{A A}$. The quantity $f_{V V}$ is sometimes confused with $f_{V}$ the 'tracer' correlation factor of vacancies. These correlation factors are quite different in concept: $f_{V V}$ refers to the collective correlation factor of the vacancies whereas $f_{V}$ refers to the correlation factor of the vacancies in the sense that each vacancy can be explicitly followed as if they were tracers (probably only physically possible in a computer simulation).

As an aside, we note that the collective correlation factor $f_{\mathrm{AA}}$ was discovered many years ago in the (interacting) lattice gas with inequivalent sites a priori [20] and many calculations of it have appeared; see the review [4]. It is not of recent origin, despite comments that appear to suggest the contrary [21]. When the atoms are non-interacting (i.e. the energy of inter-atom interactions is zero) $f_{A A}$ is trivially equal to unity. In the lattice gas the numerical values for $w_{A}$ and $f_{A A}$ depend on the particular choice of the transition probability of an atom to exchange with a neighbouring vacant site. There are an infinite number of transition probabilities that can satisfy the formal requirement 
for detailed balance. The one frequently used for studies of diffusion kinetics in lattice gases is the 'bond-breaking' transition probability [20] and is also employed below.

Eq. 11a together with Eq. 15 gives the following result for the flux $J_{A}$ of $A$ atoms:

$$
J_{A}=-\frac{z p_{A V} w_{A} a^{2} f_{A A}}{6}\left(\frac{\partial(\mu / k T)}{\partial \ln c_{A}}\right) \nabla C_{A} .
$$

Eq. 16 is seen to have the standard form of Fick's First Law with the collective diffusion coefficient $D_{A}$ being readily identified with:

$$
D_{A}=\frac{z p_{A V} w_{A} a^{2} f_{A A}}{6}\left(\frac{\partial(\mu / k T)}{\partial \ln c_{A}}\right),
$$

from which of course Eq. 3 can be readily determined.

The quantity in parentheses in Eq. 17 is usually termed the thermodynamic factor. An equivalent alternative to Eq. 17 employs the chemical potential of the $A$ species $\left(\mu_{A}\right)$ rather than the lattice gas chemical potential $\mu$. In that case, from Eq. 14, the thermodynamic factor in Eq. 17 takes the slightly different form:

$$
D_{A}=\frac{z p_{A V} w_{A} a^{2} f_{A A}}{6}\left(\frac{\partial\left(\mu_{A} / k T\right)}{c_{V} \partial \ln c_{A}}\right)
$$

At this point in our discussion, we should note the following for purely reference purposes. For non-interacting particles (apart from the usual self-blocking) it has been shown by computer simulation [22] and analytically [23] that $D_{A}$ is not a function of composition: this comes about because $f_{A A}$ equals unity as mentioned above, $w_{A}$ is constant and $p_{A V}$ is then given simply by $c_{V}$ which then cancels exactly with the thermodynamic factor (given in this one-component lattice gas case by $c_{\mathrm{V}}{ }^{-1}$ ).

Steady State Computer Simulation of the Phenomenological Coefficient. The simple cubic lattice in the computer simulation has periodic boundaries in the $y$ and $z$ directions but in the $x$ direction there are source and sink planes (one of each) of atoms. These special planes are kept at (different) constant chemical potentials using independent grand canonical ensembles. In effect these planes are in equilibrium with external reservoirs of atoms. The atoms in the lattice are permitted to diffuse in the usual way [24]. Similar to any of the previous methods, any occupied site to vacant site transition probability can be chosen that satisfies detailed balance: the 'bondbreaking' transition probability was used here in the present simulations [20,24]. Regular reequilibration of the source and sink planes is achieved by attempting several hundred exchanges with the external reservoirs in the grand canonical ensembles every time an atom leaves or arrives on a source or sink plane or their neighbouring planes. A chemical potential difference between the source and sink planes $(\Delta \mu / k T)$ of about 0.1 was found to give satisfactory results. Once a steadystate flux $J_{A}$ of $A$ atoms is established between the source and sink planes from an arbitrary starting distribution of $A$ atoms, the simulation is permitted to continue until sufficient data are accumulated for this flux and the chemical potential profile between the source and sink planes. The chemical potential profile itself is calculated at each plane by making use of Widom's [11,12] particle insertion formula for the lattice gas chemical potential $\mu$. Since the driving force $X_{i}$ is simply $-\nabla \mu$, $L_{A A}$ is obtained directly from Eq. $11 \mathrm{~b}$. We confined ourselves to temperatures well above the order/disorder temperature (repulsive interactions) and the critical unmixing temperature (attractive interactions) $|\varepsilon| / k T_{c} \leq 0.887$ at $c_{A}=0.5$ where $\varepsilon$ is the atom-atom nearest neighbour interaction energy.

We also calculated $L_{A A}$ in an equilibrium random walk calculation using the Einsteinian formula (Eq. 15), i.e. using the second direct method discussed above. In order to do this, we calculated $f_{A A}$, 
$w_{A}$ and $p_{A V}$ by following the general procedures that have been described in the literature many times, see especially the review by Murch [24] and also [4]. A simple cubic lattice of 1728 sites was used. The system was equilibrated in the petit canonical ensemble for approximately 10 jumps per atom before calculations were made based on another 40 jumps per atom. This process was repeated 10000 times in order to increase the precision of the results.

Figure 1 shows a comparison of results obtained with the new method with those obtained with the second direct method. It is clear that there is satisfactory agreement and, accordingly, the new method appears to be reliable. We have not yet investigated effects of strong chemical potential gradients. In principle, the new method might be expected to suffer from the same potential nonlinear response difficulties as the other methods except the second direct method above. Care would also need to be exercised in the preparation phase to ensure that if an ordered region happened to be entered in the spatial region between the source plane and the sink plane, that the corresponding ordered atomic distribution did not contain a non-equilibrium concentration of anti-phase boundaries. As a simplification, it is possible that in the simulation the source plane and the sink plane could simply be treated by assuming a random distribution of atoms at different compositions rather than the fully locally equilibrated ones in different grand canonical ensembles as used here. This would certainly simplify the simulation but it would, of course, corrupt the values of the chemical potentials at and near these planes. However, well away from these planes it is possible that there would be no difficulty. It is worth mentioning that diffusion problems such as the drift (and diffusion) of a tracer source in a chemical potential gradient of the one-component lattice gas seem to be amenable to this method [25]. However, at this preliminary stage of development it would appear that extensions of the method to interacting binary systems (e.g. the Ising model) are not straightforward.
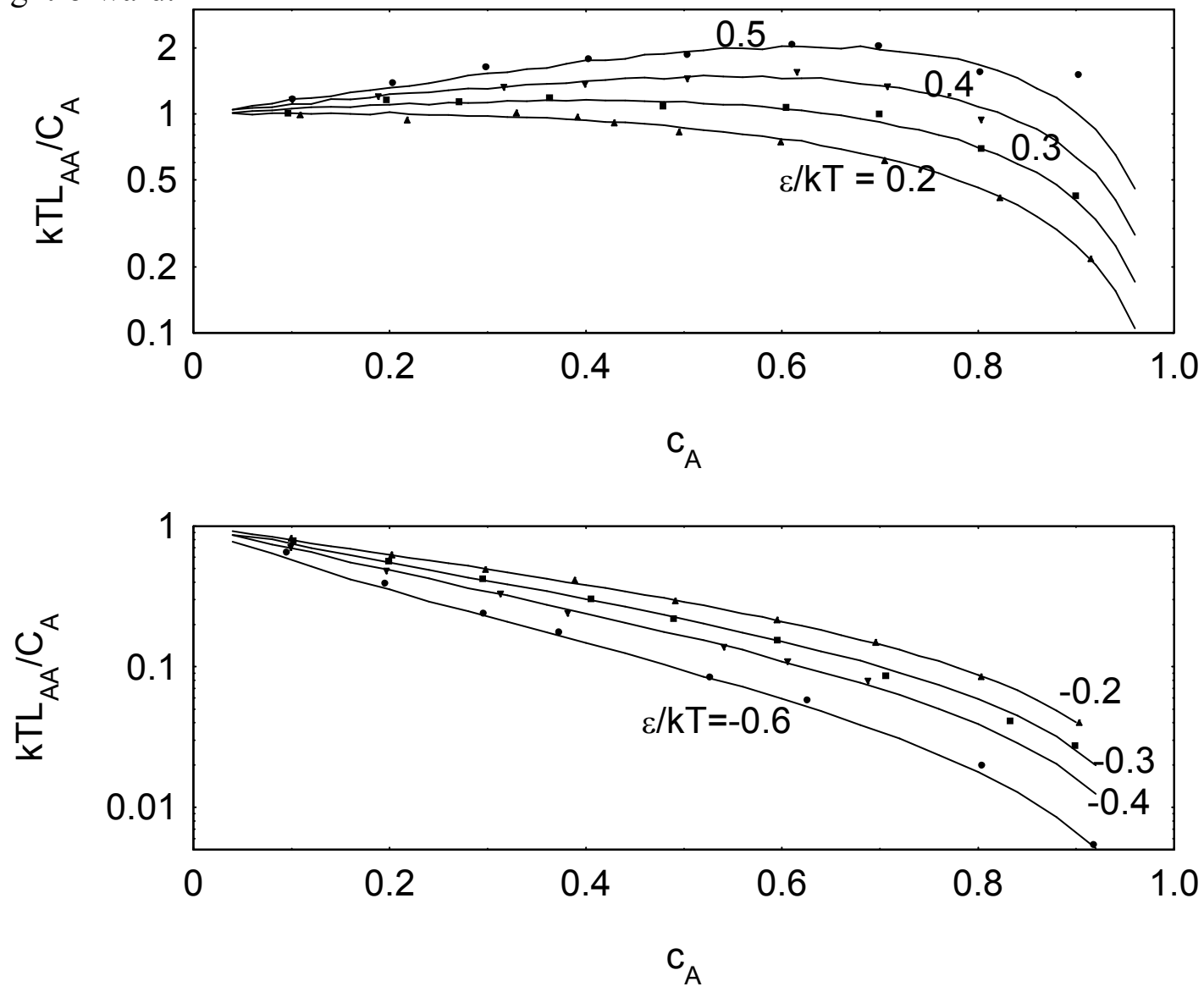

Fig. 1. Points: Monte Carlo calculation of reduced $\mathrm{L}_{\mathrm{AA}}$ by way of the Onsager Equation (Eq. 11b). Solid lines: fitted Monte Carlo calculations of reduced $\mathrm{L}_{\mathrm{AA}}$ by way of the Einsteinian Equation (Eq. $15)$. 


\section{Conclusions}

In this paper, we have addressed the Monte Carlo calculation of the Onsager phenomenological transport coefficients. First, we reviewed the principal indirect and direct Monte Carlo methods for calculating these coefficients. We provided a detailed exposition of chemical diffusion in the onecomponent lattice gas and we then proposed a new Monte Carlo method that makes use of a steady state calculation of a flux of atoms driven by a difference in chemical potential. The method was demonstrated for the simple cubic lattice gas with nearest neighbour interactions. The new method gives results in good agreement with the 'standard' direct Monte Carlo method based on the use of Einsteinian expressions for the phenomenological coefficients.

\section{Acknowledgments}

We wish to thank the Australian Research Council for its support of this research through the Discovery Grants Scheme. One of us (I.V.B.) wishes to thank the Australian Research Council for the award of a Professorial Fellowship.

\section{References}

[1] J. Philibert: Atom Movements: Diffusion and Mass Transport in Solids, (Editions de Physique, Les Ulis 1991).

[2] A.R. Allnatt and A.B. Lidiard: Atomic Transport in Solids, (Cambridge University Press, Cambridge 1993).

[3] J.R. Manning: Diffusion Kinetics for Atoms in Crystals. (Van Nostrand, Princeton, NJ 1968).

[4] G.E. Murch and J.C. Dyre: CRC Crit. Rev. Sol. St. Mat. Sci. Vol. 15 (1989), p. 345.

[5] A.R. Allnatt: J. Phys. C: Solid State Phys. Vol. 14 (1981), p. 5453 and p. 5467.

[6] C.C. Wang and S.A. Akbar: Acta Metall et Mater. Vol. 41 (1993), p. 2807.

[7] M. Nastar, V. Yu. Dobretsov and G. Martin: Phil. Mag. A Vol. 80 (2000), p. 155.

[8] J.R. Manning: Phys. Rev. B Vol. 4 (1971), p. 1111.

[9] L.K. Moleko, A.R. Allnatt and E.L. Allnatt: Phil. Mag. A Vol. 59 (1989), p. 141.

[10] T. Wichmann, K.G. Wang and K.W. Kehr: J. Phys. A: Math. Gen. Vol. 27 (1994), p. L263.

[11] B. Widom: J. Chem. Phys. Vol. 39 (1963), p. 2808.

[12] G.E. Murch and R.J. Thorn: J. Comput. Phys. Vol. 29 (1978), p. 237.

[13] M. Bowker and D.A. King: Surf. Sci. Vol. 72 (1978), p. 208.

[14] G.E. Murch and R.J. Thorn: Phil. Mag. Vol. 36 (1977), p. 529.

[15] G.E. Murch: Phil. Mag. A Vol. 46 (1982), p. 151.

[16] K.W. Kehr, K. Binder and S.M. Reulein: Phys. Rev. B Vol. 39 (1989), p. 4891.

[17] G.E. Murch and R.J. Thorn: Phil. Mag. A Vol. 39 (1979), p. 259.

[18] A.R. Allnatt: J. Phys. C Vol. 15 (1982), p. 5605.

[19] Z. Qin and G.E. Murch: Phil. Mag. A Vol. 70 (1994), 481.

[20] H. Sato and R. Kikuchi: J. Chem. Phys. Vol. 55 (1971), p. 677.

[21] P. Argyrakis and A.A. Chumak: Phys. Rev. B Vol. 66 (2002), p. 54303.

[22] G.E. Murch: Phil. Mag. A Vol. 41 (1980), p. 157

[23] R. Kutner: Phys. Lett. A, Vol. 81 (1981), p. 239.

[24] G.E. Murch: Diffusion in Crystalline Solids, ed: by G.E. Murch and A.S.Nowick (Orlando, Fl. Academic Press 1984) p 379.

[25] R.A. McKee: Phys. Rev. B Vol. 23 (1981), p. 1609. 
Diffusion in Solids - Past, Present and Future

doi:10.4028/3-908451-17-5

Calculation of Phenomenological Coefficients by Monte Carlo Computer Simulation Methods

doi:10.4028/3-908451-17-5.27 